\title{
More Peripheral Visualization of Hepatic Arteries by Using Respiratory-Triggered 3D True Steady-State Free-Precession Projection Magnetic Resonance Angiographic Sequences with Time-Spatial Labeling Inversion Pulse
}

\author{
Daisuke Tsuge, Ryohei Kuwatsuru*, Tatsuro Inoue, Yuki Yamashiro, Kazuhiro Suzuki, \\ Akihiko Shiraishi
}

Department of Radiology, Faculty of Medicine, Juntendo University, Tokyo, Japan

Email: ${ }^{*}$ da-tsuge@hotmail.co.jp

Received 2 October 2014; revised 2 November 2014; accepted 20 November 2014

Copyright (C) 2014 by authors and Scientific Research Publishing Inc.

This work is licensed under the Creative Commons Attribution International License (CC BY).

http://creativecommons.org/licenses/by/4.0/

\section{Open Access}

\begin{abstract}
Purpose: To evaluate respiratory-triggered three-dimensional (3D) true steady-state free-precession (SSFP) projection magnetic resonance angiographic sequences with time-spatial labeling inversion pulse (Time-SLIP) for visualizing the hepatic arteries and to optimize the image acquisition protocol. Materials and Methods: A 1.5-T clinical magnetic resonance imager was used to perform abdominal magnetic resonance angiography (MRA) in 25 consecutive patients before transcatheter arterial chemoembolization or surgery. We compared two selective space-labeling inversion pulse (tag pulse) patterns (Patterns I and II, oblique and parallel tag pulses, respectively). Two experienced radiologists evaluated the number of hepatic arterial branches visible on the acquired MRA images, and the results were referenced with those on images from intra-arterial digital subtraction angiography. Results: Images were acquired from all patients. The two radiologists clearly visualized branches of the left and right hepatic arteries. More peripheral hepatic arterial branches were identified in MRA images captured by using tag pulse Pattern I than in those acquired by using Pattern II $(P<0.05)$. Two patients with ascites had great numbers of peripheral hepatic arterial branches, indicating that ascites did not affect the visualization of hepatic arteries in the MRA images acquired. There was no significant difference between the scores of images from patients with Child-Pugh classifications $A$ and $B(P>0.05)$. Conclusion: Non-contrast-enhanced Time-SLIP hepatic MRA with true SSFP allowed selective visualization of peripheral hepatic ves-

${ }^{*}$ Corresponding author.

How to cite this paper: Tsuge, D., Kuwatsuru, R., Inoue, T., Yamashiro, Y., Suzuki, K. and Shiraishi, A. (2014) More Peripheral Visualization of Hepatic Arteries by Using Respiratory-Triggered 3D True Steady-State Free-Precession Projection Magnetic Resonance Angiographic Sequences with Time-Spatial Labeling Inversion Pulse. Open Journal of Radiology, 4, 314-321. http://dx.doi.org/10.4236/ojrad.2014.44041
\end{abstract}


sels.

Keywords

Hepatic Artery, Non-Contrast Magnetic Resonance Angiography, Time-Spatial Labeling Inversion Pulse, True SSFP, Angiography

\section{Introduction}

Detailed evaluation of the hepatic arteries is a necessary task before liver surgery or transcatheter arterial chemoembolization (TACE). Intra-arterial digital subtraction angiography (IADSA) is a useful technique for visualizing the hepatic arterial anatomy; however, as it is an intra-arterial procedure, it is invasive. Contrast-enhanced computed tomography (CT) and magnetic resonance angiography (MRA) are standard non-invasive procedures used to visualize the hepatic arteries [1] [2]. However, these techniques require the use of contrast agents that have the potential to cause severe adverse reactions such as anaphylactic shock, making them unsuitable for patients who have asthma, severe renal dysfunction, or a history of allergy to contrast agents. Furthermore, gadolinium-based MRA contrast agents can cause nephrogenic systemic fibrosis in patients with severe renal dysfunction [3]. Therefore, vascular imaging without contrast medium is safe and low cost modality. Non-contrast-enhanced MRA techniques are suitable for visualization of the hepatic arteries. Time-spatial labeling inversion pulse (Time-SLIP) is a form of spin labeling that uses inversion pulses to suppress the background signal before data acquisition [4]. By changing the inversion time (TI), the inflowing blood signal can be controlled and the vessel to be visualized can be selected. Recent advances in magnetic imaging technology mean that it is now possible to use multiple inversion pulses. Selective visualization of the hepatic arteries by using respiratorytriggered three-dimensional (3D) true steady-state free-precession (SSFP) combined with Time-SLIP has been reported, and the optimal TI for balancing vessel-to-liver contrast and peripheral hepatic artery visualization is $1200 \mathrm{~ms}$ [5] [6]. We consider IADSA to be the gold standard technique for visualizing hepatic arteries. Therefore, here, we scored the visualization of hepatic arteries by using non-contrast-enhanced hepatic MRA by referencing the images of IADSA. The purpose of this study is to evaluate respiratory-triggered three-dimensional (3D) true steady-state free-procession (SSFP) projection magnetic resonance angiographic sequences with timespatial labeling inversion pulse (Time-SLIP) for visualizing the hepatic arteries and to optimize the image acquisition protocol.

\section{Materials and Methods}

\subsection{Subjects}

From August 2011 to July 2012, 25 consecutive patients (21 men and 4 women; mean age, 73.1 years; range, 3 82 years; Child-Pugh classification A and B, $n=15$ and $n=10$, respectively) were recruited to the study. Twenty-three examinations were conducted within the 2 days before the patients underwent TACE for hepatocellular carcinoma. One case was performed about three weeks before TACE, and the other case did not undergo TACE because of a history of allergy to contrast agents. This study obtained ethical approval from our institutional review board. All source images were transferred to an independent workstation and subjected to multiplanar reconstruction and maximum intensity projection (SYNAPSE ${ }^{\circledR}$ Enterprise-PACS FUJIFILM, Japan). Images were analyzed retrospectively.

\subsection{MRA}

All examinations were performed on a 1.5-T MR imager (EXCELART ${ }^{\circledR}$ Vantage Powered by Atlas, Toshiba Medical Systems, Tokyo, Japan) with the patient supine. The imager equipped with a pair of phased array coils containing 12 active coil elements connected to 12 receiver channels which were placed at the front and back of the abdomen. The parameters used were as follows: TR, $4300 \mathrm{~ms}$; TE, $2.2 \mathrm{~ms}$; flip angle, $120^{\circ}$; slice thickness, 3 $\mathrm{mm}$; FOV , $400 \times 400 \mathrm{~mm}$; matrix, $256 \times 256$; STIR TI, $1200 \mathrm{~ms}$; respiration rate, $4000 \mathrm{~ms}$. 


\subsection{Time-SLIP}

Two different Time-SLIP tag pulse patterns were used (Figure 1): Pattern I comprised three tag pulses. Two of the tag pulses were oblique. The 1st tag pulse was placed such that the superior border was parallel to the upper edge of the liver and celiac trunk to suppress the background tissue, and the 2 nd tag pulse was placed caudal to $1 \mathrm{st}$ tag pulse to suppress the flow signal from the inferior vena cava. There was a distance of about $1 \mathrm{~cm}$ space between 1st and 2nd tag pulses, not so as to pile up each other's pulses. The 3rd tag pulse was placed vertically to suppress the flow signal from the splenic vein. Pattern II comprised two oblique pulse tags both parallel to the diaphragm. Both patterns of tag pulse covered the splenic vein and superior mesenteric vein to suppress the signal from blood flowing into the liver. The most important difference between the two types of tag pulse was the starting point of the signal from the inflow through the aorta. Three cases were performed only tag pulse Pattern I. The acquisition time of each pattern's scan time was from three to five minutes.

\subsection{Hepatic Arterial Scoring}

Two radiologists (with 8 and 22 years of experience) evaluated the number of hepatic arterial branches visible in the acquired images. Both the left and right hepatic arteries were examined, because the study included patients who had undergone partial resection of the hepatic lobe (left or right). Angiographic results of 24 cases were used as standards for comparison. One case was evaluated by only MRA images. The images of angiography were acquired by injecting contrast agent from celiac artery. MRA Images were scored in accordance with the number of arterial branches visible (Figure 2). For example, a score of 3 was given if the sub-branches of a hepatic artery could be identified. A score of 4 was the case that third-order branch of right or left hepatic artery was visualized, and a score of 5 was forth-order branch was visualized. Both radiologists were blinded to the scan conditions and the images were evaluated in random order. When the scores from the radiologists differed, a discussion was held until an agreement was reached.

\subsection{Statistical Analysis}

Statistical analyses were performed by using a commercially available software package (SPSS, Chicago, IL). Paired t test was performed, and a $P$-value of less than 0.05 was considered to indicate a statistically significant difference.

\section{Results}

Images were acquired from all patients and no motion artifacts were seen. The total imaging time for each

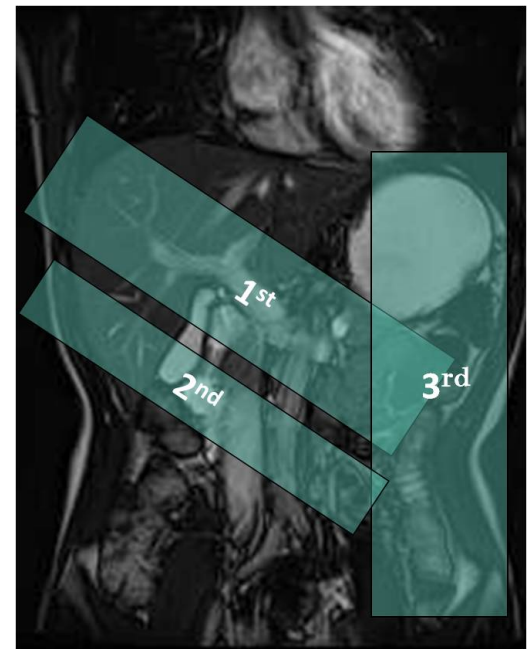

(a)

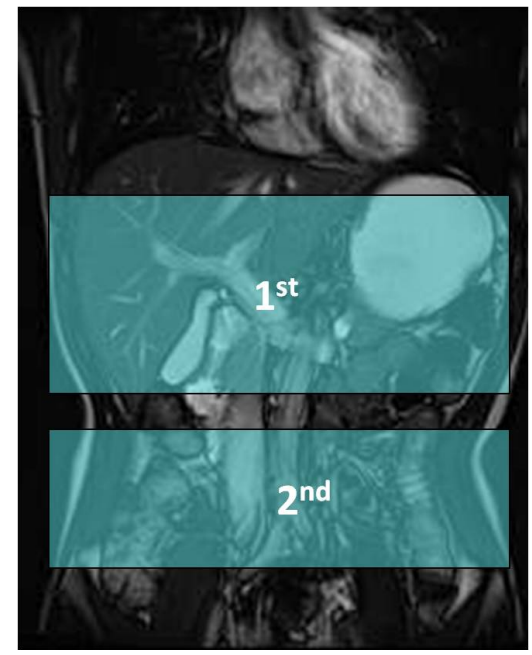

(b)

Figure 1. Tag pulse patterns used: (a) Pattern I: two oblique (1st and 2nd) and one vertical tag pulses (3rd); (b) Pattern II: two parallel tag pulses (1st and 2nd). 
Time-SLIP was approximately $10 \mathrm{~min}$. Twenty four patients experienced TACE and we established the images of intra-arterial angiogram as the gold standard. After referencing the angiogram, the two radiologists evaluated the hepatic MRA images. More peripheral branches were identified in images captured by using tag pulse Pattern I than in images captured by using Pattern II $(P<0.05)$. The highest score of 5 (meaning that the fourthorder branches of the artery could be detected) was achieved in only one case, of visualization of the right hepatic artery with tag pulse Pattern I, although this patient did have ascites (Table 1 and Figure 3). In spite of better quality images of Pattern I, three left hepatic arteries (LHA) and two right hepatic arteries (RHA) were not visualized. Their average age was 80.0 years. Three of five patients breathed irregularly and did not synchronize the respiratory-triggered scan. Aging and irregular breathing might be defectiveness factor. There were no significant differences between the scores of images from patients with Child-Pugh classifications A and B for either hepatic artery $(P>0.05)$ (Table 2$)$. Normal hepatic arterial anatomy was seen in 21 patients and anatomical variations were seen in four patients: two patients had a replaced right hepatic artery arising from the superior mesenteric artery, and two had an aberrant left hepatic artery arising from the left gastric artery. Both radiologists identified the aberrant arteries before angiography was performed (Figure 4 and Figure 5). The vascular variants were coincident with the results of angiography (IADSA).

\section{Discussion}

Multi-detector-row 3D CT angiography provides excellent visualization of vascular details, and owing to its accessibility and brief scan time it is now a commonly used technique for assessing liver anatomy [7] [8]. Moreover, 320-row CT 4D DSA can accurately visualize normal anatomy and variation of hepatic artery, dynamically display tumor staining and reproducibly delineate the three-dimension relationship between tumor and blood vessels [9]. However, CT scans expose the patient to ionizing radiation and intravenous contrast agents that may cause serious adverse events such as anaphylactic shock. Contrast-enhanced MRA is a useful method for evaluating

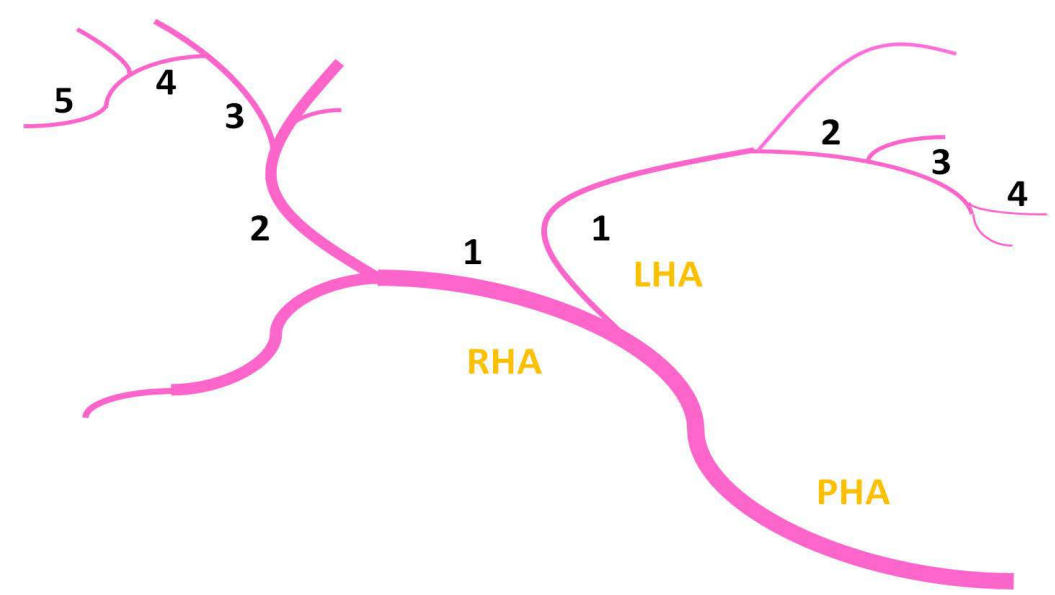

Figure 2. Images were scored as shown and compared with angiographic results. Right and left arteries were evaluated. LHA: left hepatic artery; PHA: proper hepatic artery; RHA: right hepatic artery.

Table 1. Hepatic artery scores for tag pulse Patterns I and II.

\begin{tabular}{cccccccc}
\hline & & \multicolumn{4}{c}{ Hepatic Artery Score } \\
Hepatic Artery & Tag Pulse Pattern & 0 & 1 & 2 & 3 & 4 & 5 \\
\cline { 2 - 6 } LHA & Pattern I $(\mathrm{n}=23)$ & 3 & 8 & 9 & 2 & 1 & 0 \\
LHA & Pattern II $(\mathrm{n}=21)$ & 9 & 6 & 5 & 1 & 0 & 0 \\
RHA & Pattern I $(\mathrm{n}=25)$ & 2 & 3 & 6 & 7 & 6 & 1 \\
RHA & Pattern II $(\mathrm{n}=22)$ & 7 & 3 & 5 & 5 & 2 & 0 \\
\hline
\end{tabular}

LHA: left hepatic artery; RHA: right hepatic artery. 
Table 2. Differences in liver function: Child-Pugh (CP) classifications A and B (tag pulse Pattern I).

\begin{tabular}{cccccccc}
\hline & \multicolumn{5}{c}{ Hepatic Artery Score } \\
\cline { 2 - 6 } Hepatic Artery & Tag Pulse Pattern & 0 & 1 & 2 & 3 & 4 \\
\hline LHA & CPA $(\mathrm{n}=13)$ & 1 & 5 & 4 & 2 & 1 & 0 \\
LHA & $\mathrm{CPB}(\mathrm{n}=10)$ & 2 & 3 & 5 & 0 & 0 & 0 \\
RHA & $\mathrm{CPA}(\mathrm{n}=15)$ & 2 & 1 & 2 & 6 & 3 & 1 \\
RHA & $\mathrm{CPB}(\mathrm{n}=10)$ & 0 & 2 & 4 & 1 & 3 & 0 \\
\hline
\end{tabular}

CPA: Child-Pugh classification A; CPB: Child-Pugh classification B.

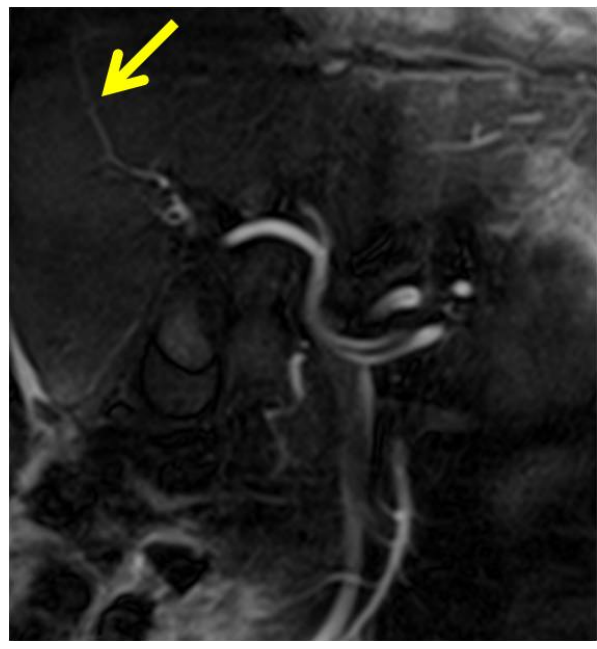

(a)

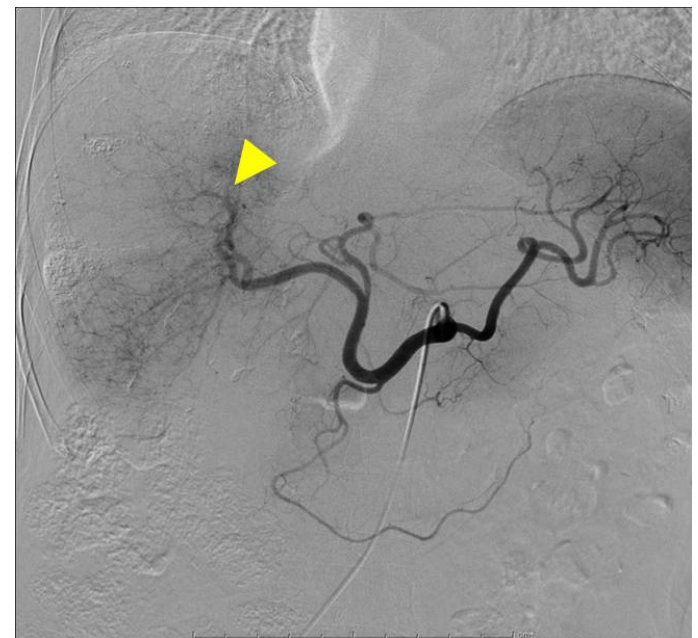

(b)

Figure 3. (a) MRA image with the highest score (tag pulse Pattern I). The most peripheral hepatic arteries are identifiable in this image (arrow), although this patient did have ascites. (b) Angiography in the patient shown in (a). The contrast agent was injected into the celiac artery and showing the right hepatic artery (arrow head).

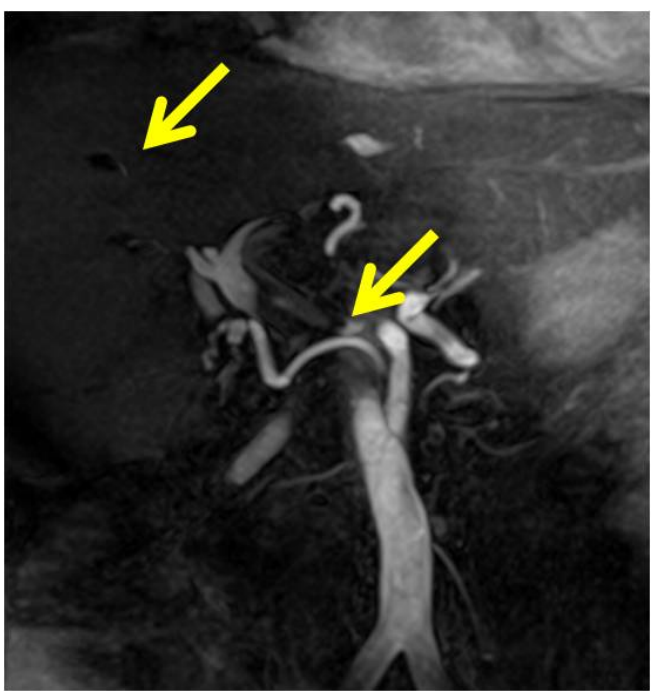

(a)

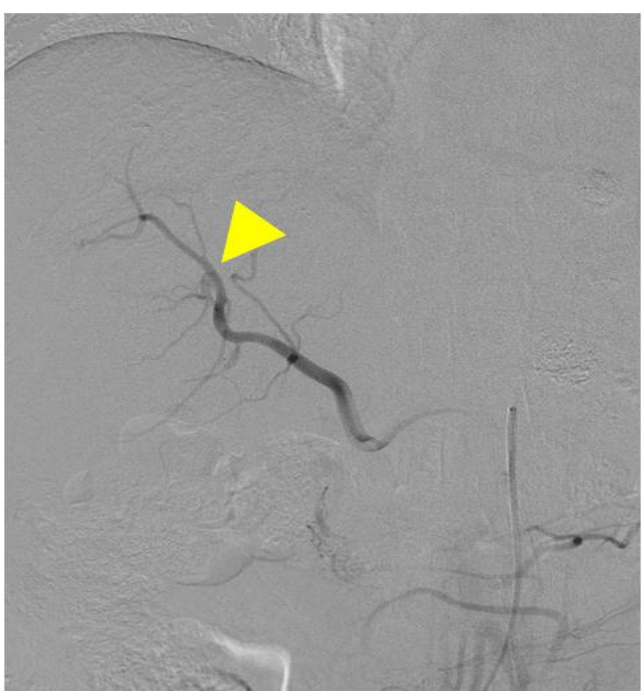

(b)

Figure 4. (a) MRA image of replaced right hepatic artery arising from the superior mesenteric artery (arrows). (b) Angiography of the vessel shown in (a) (arrow head). 


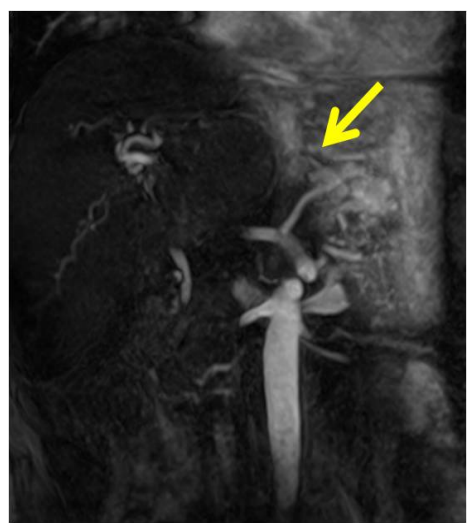

(a)

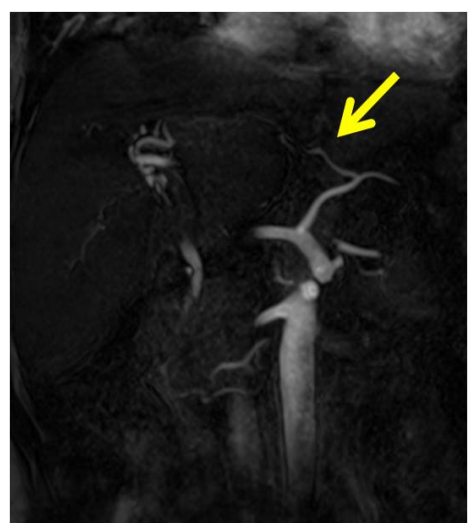

(b)

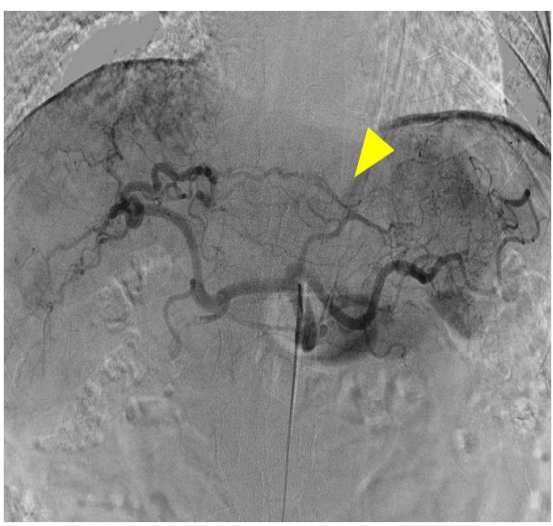

(c)

Figure 5. MRA images captured by using (a) tag pulse Pattern I and (b) tag pulse Pattern II. (c) Angiography of the vessels shown in (a) and (b). Aberrant left hepatic artery arising from the left gastric artery (arrows) is clearly visualized on both MRA images ((a) and (b)). Tag pulse Pattern II provides a clearer depiction of the branch of the aberrant left hepatic artery (arrow) owing to greater suppression of the background signal. (c) Angiography showing the aberrant left hepatic artery arising from the left gastric artery (arrow head).

vascular disease without the need to expose the patient to radiation [10] [11]. However, patients with moderate to severe renal dysfunction may develop a serious adverse reaction called nephrogenic systemic fibrosis in response to gadolinium-based MR contrast agents. This adverse event is rare but non-reversible. Other adverse events such as anaphylactic shock are sometimes fatal. Therefore, non-contrast techniques are required for visualizing the hepatic vessels in these patients.

Previous reports have described the selective visualization of hepatic arteries without the need for an exogenous contrast agent by using a combination of respiratory-triggered 3D true SSFP sequences and Time-SLIP [5] [6]. Recently, Shimada et al. demonstrated that fat suppression by means of short-tau inversion recovery provides better background signal suppression than chemical shift selective pulse [5]. The most important factor for clearly visualizing hepatic arteries with non-contrast-enhanced hepatic MRA is TI. A short TI (800 or $1000 \mathrm{ms)}$ favorably suppresses the background signal, but the inflow signal is weak and therefore the images obtained are unsuitable for peripheral vessel visualization [5]. A longer TI (1400 ms) shows more peripheral arterial flow, but the vessel-to-liver contrast deteriorates because of background signal recovery. An intermediate TI of $1200 \mathrm{~ms}$ allows the visualization of more peripheral hepatic arteries and suppresses the flow signal of the splenic vein, superior mesenteric vein, and inferior vena cava [5] [6]. A TI of $1200 \mathrm{~ms}$ gives the optimal balance between vessel-to-liver contrast and peripheral hepatic artery visualization [5], and therefore was the TI that we used here. We used short-tau inversion recovery not only to obtain complete fat suppression but also to improve image quality by suppressing signals from the surrounding tissues (liver parenchyma, gastrointestinal tract, and gallbladder) [6] [12] [13]. We also examined different patterns of inversion pulse placement. Placement of the inversion pulses closer to the start point of the inflowing arterial signal provided better visualization of the peripheral vessels, which is why the hepatic arteries were better visualized with tag pulse Pattern I than with Pattern II. The main difference between the two tag pulse patterns was where the inflowing blood signal from the aorta, superior mesenteric artery, and several veins (such as the inferior vena cava, splenic vein, and portal vein) began. The superior border of tag pulse Pattern I was placed near the celiac artery, whereas that of Pattern II was placed at the descending aorta at a level of the heart inferior border (Figure 1). This means that the inflowing signal in Pattern I began at the celiac trunk, whereas that in Pattern II was nearer the descending aorta. This difference in where the inflow began influenced the visualization of the peripheral hepatic arteries (Figure 6). However, the disadvantage of tag pulse Pattern I was seen in the poor visualization of one case of aberrant left hepatic artery arising from the left gastric artery (Figure 4). The image was of poor quality because the branch of this vessel was outside the tag pulse pattern, so the background signal was not suppressed.

We expected the flow in the hepatic arteries of patients with liver dysfunction to be slow and poorly visualized because of intrahepatic vascular increased pressure and resistance [14]. However, the scores of images from patients with Child-Pugh classifications A and B were not significantly different $(P>0.05)$. Patients with Child-Pugh classification $C$ were not enrolled in the study: these patients have substantial liver dysfunction and 


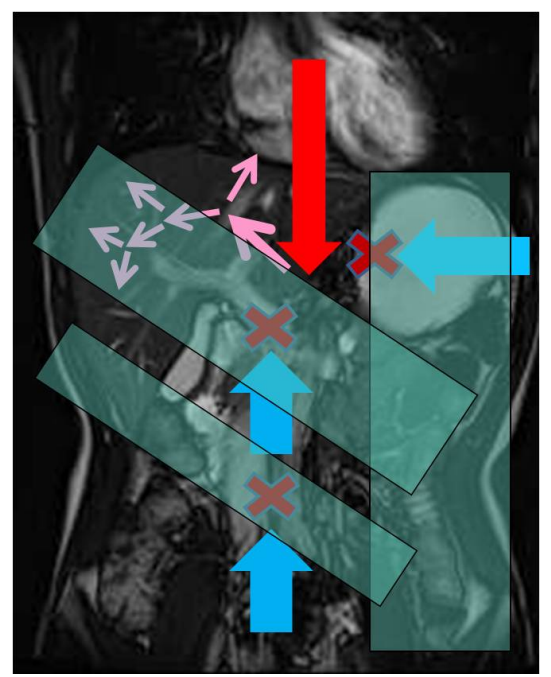

(a)

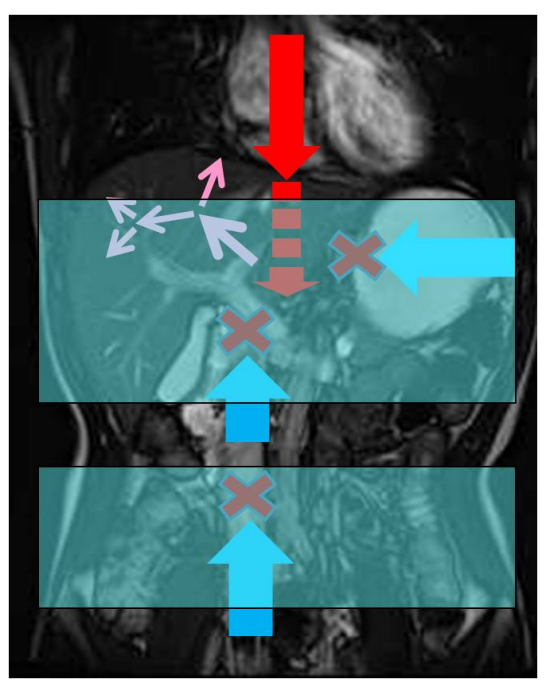

(b)

Figure 6. (a) Tag pulse Pattern I; (b) Tag pulse Pattern II. Two different patterns of tag pulse give different depictions of the hepatic artery. Red arrow means arterial flow, and light blue flow means venous flow. Pink arrow is visualized hepatic arterial flow acquired by MRA images. More peripheral arteries can be visualized with tag pulse Pattern I because the start point of the inflowing blood signal of the aorta is different. The start point of Pattern I is the celiac trunk, and Pattern II is the descending aorta at a level of the cardiac inferior border. More peripheral start point can visualize more hepatic arteries.

are in overall poor health and are therefore not candidates for TACE. One important point to note regarding our results is that patients with ascites had high scores, indicating that ascites did not influence the visualization of hepatic arteries with Time-SLIP MRA.

\section{Conclusion}

This is the report to evaluate non-contrast-enhanced hepatic MRA by referencing angiography for the visualization of hepatic arteries. Non-contrast-enhanced hepatic MRA with Time-SLIP is a safe and effective method for visualizing hepatic arterial anatomy before angiography or surgery.

\section{References}

[1] Lee, M.W., Lee, J.M., Lee, J.Y., et al. (2007) Preoperative Evaluation of Hepatic Arterial and Portal Venous Anatomy Using the Time Resolved Echo-Shared MR Angiographic Technique in Liver Donors. European Journal of Radiology, 17, 1074-1080. http://dx.doi.org/10.1007/s00330-006-0447-3

[2] Heilmaier, C., Sutter, R., Lutz, A.M., et al. (2007) Mapping of Hepatic Vascular Anatomy: Dynamic Contrast-Enhanced Parallel MR Imaging Compared with 64 Detector Row CT. Radiology, 245, 872-880. http://dx.doi.org/10.1148/radiol.2453062103

[3] Buhaescu, I. and Izzedine, H. (2008) Gadolinium-Induced Nephrotoxicity. International Journal of Clinical Practice, 62, 1113-1118. http://dx.doi.org/10.1111/j.1742-1241.2007.01582.x

[4] Garcia, D.M., Duhamel, G. and Alsop, D.C. (2005) Efficiency of Inversion Pulses for Background Suppressed Arterial Spin Labeling. Magnetic Resonance in Medicine, 54, 366-372. http://dx.doi.org/10.1002/mrm.20556

[5] Shimada, K., Isoda, H., Okada, T., et al. (2009) Non-Contrast-Enhanced Hepatic MR Angiography with True SteadyState Free-Precession and Time Spatial Labeling Inversion Pulse: Optimization of the Technique and Preliminary Results. European Journal of Radiology, 70, 111-117. http://dx.doi.org/10.1016/j.ejrad.2007.12.010

[6] Shimada, K., Isoda, H., Okada, T., et al. (2011) Non-Contrast-Enhanced Hepatic MR Angiography: Do Two-Dimensional Parallel Imaging and Short Tau Inversion Recovery Methods Shorten Acquisition Time without Image Quality Deterioration? European Journal of Radiology, 77, 137-142. http://dx.doi.org/10.1016/j.ejrad.2009.05.051

[7] Kang, H.K., Jeong, Y.Y., Choi, J.H., et al. (2002) Three-Dimensional Multidetector Row CT Portal Venography in the Evaluation of Porto-Systemic Collateral Vessels in Liver Cirrhosis. Radiographics, 22, 1052-1061. 
http://dx.doi.org/10.1148/radiographics.22.5.g02se011053

[8] Yamashita, K., Sakuramoto, S., Mieno, H., et al. (2014) Preoperative Dual-Phase 3D CT Angiography Assessment of the Right Hepatic Artery before Gastrectomy. Surgery Today, 44, 1912-1919. http://dx.doi.org/10.1007/s00595-014-0858-8

[9] Wang, J., Liao, B.H., Liu, J.J., et al. (2013) Clinical Application of 320-Row Computed Tomography 4D Digital Subtraction Angiography in Hepatocellular Carcinoma. Zhonghua Yi Xue Za Zhi, 93, 3294-3296.

[10] Kreft, B., Strunk, H., Flacke, S., et al. (2000) Detection of Thrombosis in the Portal Venous System: Comparison of Contrast-Enhanced MR Angiography. Radiology, 216, 86-92. http://dx.doi.org/10.1148/radiology.216.1.r00j12386

[11] Aslam, R., Yeh, B.M., Yee, J., et al. (2010) MR Imaging Evaluation of the Hepatic Vasculature. Magnetic Resonance Imaging Clinics of North America, 18, 512-523. http://dx.doi.org/10.1016/j.mric.2010.08.005

[12] Takahara, T., Imai, Y., Yamashita, T., et al. (2004) Diffusion Weighted Whole Body Imaging with Background Body Signal Suppression (DWIBS): Technical Improvement Using Free Breathing, STIR and High Resolution 3D Display. Radiation Medicine, 22, 275-282.

[13] Shonai, T., Takahashi, T., Ikeguchi, H., et al. (2009) Improved Arterial Visibility Using Short-Tau Inversion-Recovery (STIR) Fat Suppression in Non Contrast-Enhanced Time-Spatial Labeling Inversion Pulse (Time-SLIP) Renal MR Angiography (MRA). Journal of Magnetic Resonance Imaging, 29, 1471-1477. http://dx.doi.org/10.1002/jmri.21792

[14] Yasuko, I., Vijay, S. and Don, C.R. (2014) Vascular Pathobiology in Chronic Liver Disease and Cirrhosis-Current Status and Future Directions. Journal of Hepatology, 61, 912-914. 
Scientific Research Publishing (SCIRP) is one of the largest Open Access journal publishers. It is currently publishing more than 200 open access, online, peer-reviewed journals covering a wide range of academic disciplines. SCIRP serves the worldwide academic communities and contributes to the progress and application of science with its publication.

Other selected journals from SCIRP are listed as below. Submit your manuscript to us via either submit@scirp.org or Online Submission Portal.
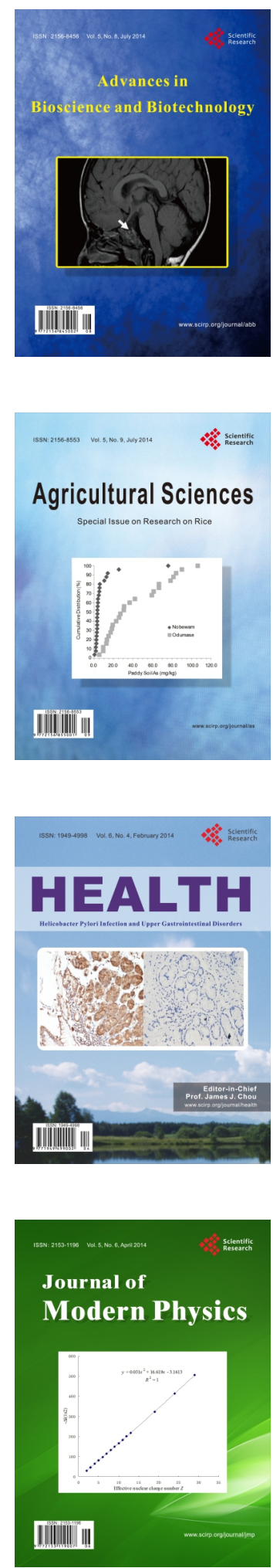
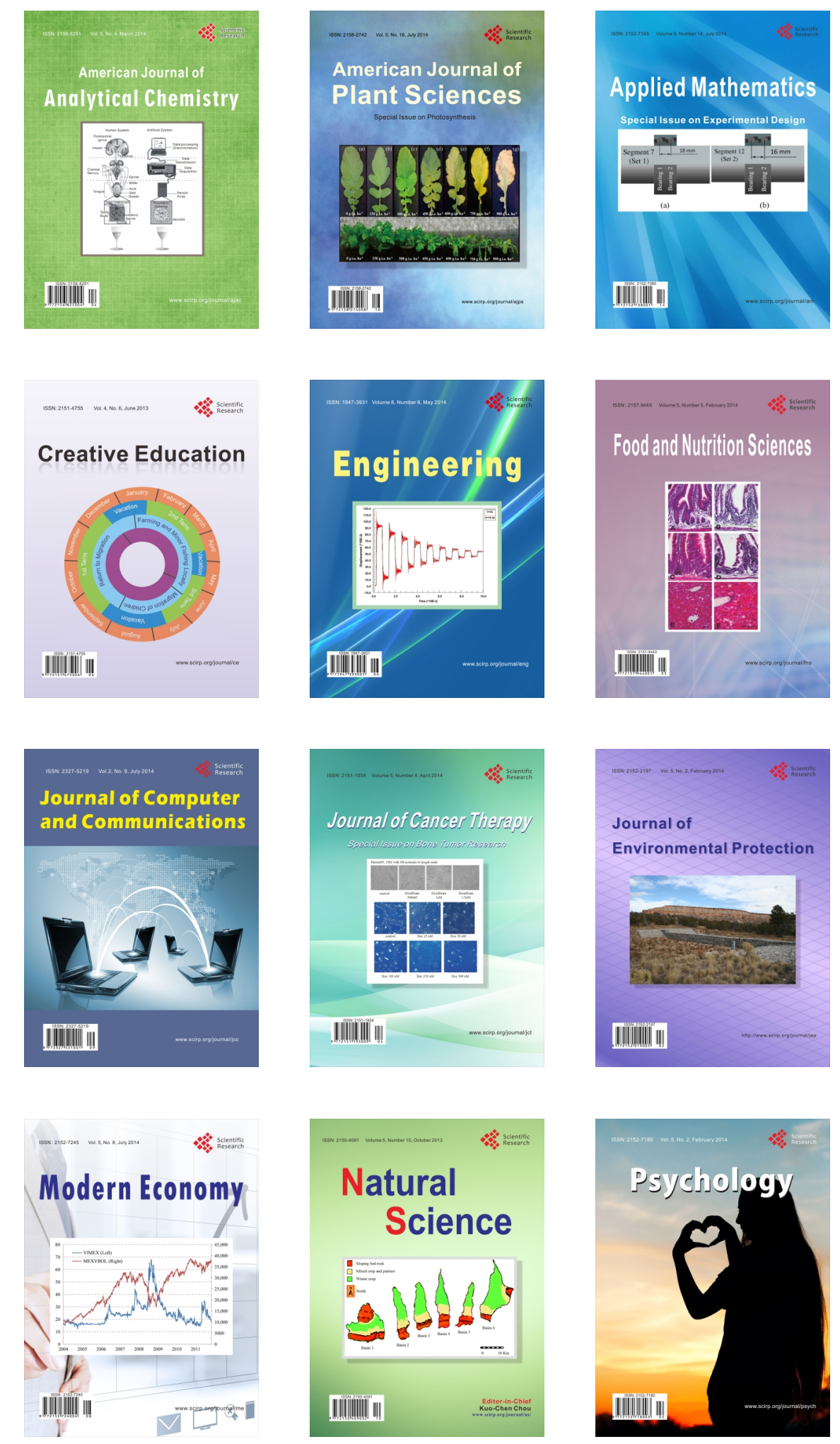